\title{
Oral and anal high-risk human papilloma virus infection in HIV-positive men who have sex with men over a 24-month longitudinal study: complexity and vaccine implications
}

\author{
Saverio Giuseppe Parisi ${ }^{*}$, Monica Basso $^{1 *}$ (D), Renzo Scaggiante ${ }^{2}$, Samantha Andreis ${ }^{1}$, Carlo Mengoli ${ }^{1}$,
} Mario Cruciani ${ }^{3}$, Claudia Del Vecchio $^{1}$, Nicola Menegotto', Daniela Zago ${ }^{1}$, Loredana Sarmati ${ }^{4}$, Massimo Andreoni ${ }^{4}$ and Giorgio Palù ${ }^{1}$

\begin{abstract}
Background: Few studies focused on longitudinal modifications over time of high-risk HPV (HR-HPV) at anal and oral sites in HIV+ men who have sex with men (MSM).

Methods: We described patterns and longitudinal changes of HR-HPV detection and the prevalence of HR-HPV covered by the nonavalent HPV vaccine (vax-HPV) at oral and anal sites in 165 HIV+ MSM followed in an Italian hospital. The samples were collected at baseline and after 24 months (follow-up). The presence of HPV was investigated with Inno-LiPA HPV Genotyping Extra II.
\end{abstract}

Results: Median age was 44 years (IQR 36-53), median CD4+ cell count at nadir was 312 cells $/ \mathrm{mm}^{3}$ (IQR 187-450). A total of 120 subjects (72.7\%) were receiving successful antiretroviral therapy (ART). At baseline and follow-up, the frequency of HR-HPV was significantly higher in the anal site (65.4\% vs 9.4 and $62.4 \%$ vs $6.8 \%$, respectively). Only $2.9 \%$ of subjects were persistently HR-HPV negative at both sites. All oral HR-HPV were single at baseline vs $54.6 \%$ at baseline at the anal site $(p=0.005)$, and all oral HR-HPV were single at follow-up vs $54.4 \%$ at anal site at follow-up $(p=0.002)$. The lowest rate of concordance between the oral and anal results was found for HR-HPV detection; almost all HR-HPV positive results at both anal and oral sites had different HR-HPV.The most frequent HR-HPV in anal swabs at baseline and follow-up were HPV-16 and HPV-52.At follow-up at anal site, 37.5\% of patients had different HR-HPV genotypes respect to baseline, 28.8\% of subjects with 1 HR-HPV at baseline had an increased number of HR-HPV, and patients on ART showed a lower frequency of confirmed anal HR-HPV detection than untreated patients $(p=0.03$ ) over time. Additionally,54.6 and $50.5 \%$ of patients had only HR-vax-HPV at anal site at baseline and follow-up, respectively; 15.2\% had only HR-vax-HPV at baseline and follow-up.

Conclusions: We believe that it is important testing multiple sites over time in HIV-positive MSM. ART seems to protect men from anal HR-HPV confirmed detection. Vaccination programmes could reduce the number of HR-HPV genotypes at anal site and the risk of the first HR-HPV acquisition at the oral site.

Keywords: High risk HPV, Men who have sex with men, Anal, Oral, HIV infection

\footnotetext{
* Correspondence: saverio.parisi@unipd.it; monica.basso@unipd.it

${ }^{1}$ Department of Molecular Medicine, University of Padova, Via Gabelli 63,

35100 Padova, Italy

Full list of author information is available at the end of the article
}

(c) The Author(s). 2019 Open Access This article is distributed under the terms of the Creative Commons Attribution 4.0 International License (http://creativecommons.org/licenses/by/4.0/) which permits unrestricted use, distribution, and reproduction in any medium, provided you give appropriate credit to the original author(s) and the source, provide a link to the Creative Commons license, and indicate if changes were made. The Creative Commons Public Domain Dedication waiver (http://creativecommons.org/publicdomain/zero/1.0/) applies to the data made available in this article, unless otherwise stated. 


\section{Background}

Patients with HIV infection are more likely to experience coinfection with human papillomavirus (HPV) compared to HIV negative subjects. Within the HIV positive population, over $80 \%$ of men who have sex with men (MSM) were HPV-positive in the anal canal, being higher than $80 \%$ and with a high frequency (ranging from 70 to $90 \%$ ) of high risk HPV (HR-HPV) in most studies $[1,2]$. The rates of oral mucosa infection are lower: a value of $28.9 \%$ for the pooled prevalence of any type of HPV and of $16.5 \%$ for the pooled prevalence of HR-HPV was reported in a random effects meta-analysis and meta-regression on prevalence estimated on 11 studies including 1886 HIV positive MSM [3]. All studies but one [4] explored factors associated with any type of HPV infection; in the multivariate analyses, median age remained a significant contributor to the heterogeneity of any HPV prevalence, after adjusting for HIV status.

The persistence of anal HPV DNA infection plays a central role in carcinogenesis. Marra et al. [5] reported that only anal HPV DNA persistence was independently associated with anal high-grade squamous intraepithelial lesions both in general and by concordant causative HPV-type in a cohort of HIV-positive MSM with a mean nadir CD4 T-cell count of $245 \pm 134$ cells $/ \mathrm{mm}^{3}$. Limited evidence-based data on the independent role of longterm HPV DNA positivity on the incidence of head and neck squamous cell carcinoma in HIV+ MSM are available [6].

However, negative HPV detection does not imply definite clearance of the infection because the patient's immune system may be able to control the infection below the detection limit of available HPV assays [7]. Furthermore, testing different sites of the same subject can show different results that are not associated with one another [8], and HPV-DNA positivity rates are higher in HIV positive MSM with respect to HIV negative men at external genital sites, the anal canal and the oral cavity [9]. Therefore, longitudinal studies including the testing of at least 2 anatomic sites is useful to properly identify the HPV status of patients.

The aim of this work was to analyze the numerosity and the confirmed detection of oral and anal HPV infection over a 24-month period in a cohort of HIV-positive MSM; we focused on pattern and longitudinal changes in HR-HPV detection and the prevalence of HPV genotypes covered by the nonavalent HPV vaccine.

\section{Methods}

\section{Study design}

HIV-positive MSM treated as outpatients at the Infectious Disease Unit of Padova University Hospital (Italy) from January 2013 to December 2013 were consecutively invited to take part to this study.
The following inclusion criteria were applied: 1) aged $>18$ years; 2) no cutaneous and/or mucosal lesion due to HPV infection at study entry and in the past; 3 ) no previous $\mathrm{HPV}$ vaccination; and 4) able to make the decision to sign the informed consent. Anal HPV testing was included in their clinical workflow: they were asked to give the consent for testing also oral mucosa and for the use of their blinded data. No payment was provided to the subjects.

HPV was tested in oral and anal swabs. All enrolled patients were evaluated by a physician and the samples were collected on two different visits separated by a 24month interval (the maximum spread was 2 weeks before or after the deadline). The study time corresponding to the first sampling was defined as baseline and the time corresponding to the second sampling was defined as follow-up.

The treating physician decided to start antiviral therapy or to left the patients untreated as prescribed by international guidelines that were current when the study started [10]. The subjects were defined with undetectable plasma HIV viremia when no HIV RNA determination higher than 50 copies/ml was obtained during the entire study period; the detection of one viral blip (defined as a single plasma HIV RNA value between 50 and 500 copies/ml followed by a viral load not detectable) a year was acceptable [11]. The definition of detectable HIV viremia included any other result [12].

The variables studied at baseline were age in years at baseline, the achievement of plasma HIV RNA suppression throughout the study duration and CD4 lymphocyte count at nadir $(\mathrm{cells} / \mu \mathrm{l})$.

The Ethics Committee of Padova University Hospital (prot. 2606-12P) approved this study, which was made according to the Declaration of Helsinki and local legislation. The patients included in the study gave written informed consent to all procedures and they agreed with the anonymous use of their data for scientific aims and publication.

\section{Specimen testing}

At baseline and at follow-up, anal Dacron swab PBS (phosphate-buffered saline)-moistened was inserted for $3 \mathrm{~cm}$ length into the anal canal, then the scraping of anal walls was made, rotating clockwise and anticlockwise three times each way. Oropharyngeal mucosa was scraped with strength to obtain oral samples by rotating twice clockwise and twice anticlockwise with another swab. After, the samples were agitated in $1.5 \mathrm{~mL}$ of $1 \times$ PBS. The samples were stored at $4{ }^{\circ} \mathrm{C}$ and analyzed after no more than $4 \mathrm{~h}$. The QIAamp DNA Mini Kit (Qiagen, $\mathrm{GmbH}$, Hilden, Germany) was used for total DNA extraction: after, primers $\mathrm{PC} 04$ and GH20 (which target a 
268-bp fragment of beta-globin) were first used. The samples were always processed and stored separately.

No further analysis was performed on samples negative for beta-globin. ExoSAP-IT (USB Corporation, Cleveland, $\mathrm{OH}$ ) was used to purify the DNA and InnoLiPA HPV Genotyping Extra II (Fujirebio Europe N.V., Gent, Belgium) [13] was the method chosen to investigate the presence of HPV DNA. All HPV types included in INNO-Lipa assay were tested. We did not include the detailed data in the study because we focused on HRHPV. The HPV genotype risk level was categorized according to the criteria defined by the International Agency for Research on Cancer: HR-HPV types with sufficient evidence for causing cancer include HPV-16, $-18,-31,-33,-35,-39,-45,-51,-52,-56,-58$ and - 59 [14]. Overall, other HPV genotypes and non typeable HPV were considered not high risk (non-HR).

\section{Statistical analysis}

The statistical analysis was performed on all subjects whose valid results were available and on the subgroup of subjects who had HPV infection due to genotypes covered by the nonavalent vaccine (HPV types 16, 18, 31, 33, 45, 52 and 58) [15]. The HPV testing results were defined as HR when one or more HR genotypes were detected, regardless of coinfection with non-HR; the results were defined as non-HR when only non-HR-HPV genotypes were identified in the sample.

Continuous variables were expressed as the median and interquartile range whereas categorical variables were indicated as the absolute number and frequency (\%). The Mann-Whitney U test, the chi-squared test and Fisher's exact test were employed as appropriate.

The significance of the prevalence change from baseline to follow-up was estimated by Pearson's chi-square test or by Fisher's exact test, as appropriate.

$P$ values $<0.05$ were considered to be significant.

\section{Results}

A total of 171 HIV+ MSM responded to the inclusion criteria: 4 subjects had not CD4+ cell count available and 2 subjects refused to provide informed consent, so $165 \mathrm{HIV}$ + MSM were included in the study. The median age was 44 years (IQR 36-53 years), and the median CD4+ cell count at nadir was 312 cells $/ \mathrm{mm}^{3}$ (IQR $187-450$ cells/ $\mathrm{mm}^{3}$ ). Further, 120 subjects $(72.7 \%)$ were receiving successful ART, and most patients were treated with a protease inhibitor as the third drug (91 of 120, 75.8\%).

Valid anal samples were obtained from all patients at baseline and follow-up, whereas valid oral samples were obtained from 106 (64.2\%) patients at baseline and from $162(98.2 \%)$ patients at follow-up. Invalid oral samples were not analyzed if they were found negative for beta globin, possibly because of low cellularity. The overall prevalence of HPV (both HR-HPV and non-HR-HPV) in the anal specimens was $89.1 \%$ (147/165 patients) at baseline and $89.7 \%$ (148/165 patients) at follow-up, and it was significantly lower in the oral specimens $(28.3 \%$, $30 / 106$ patients at baseline and 22.8\%, 37/162 patients, at follow-up, $p<0.0001) \mathrm{HPV}$ detection was confirmed in $12 / 103(11.6 \%)$ oral samples and in $135 / 165$ anal samples $(81.8 \%)$.

Anal and oral HR-HPV detection at baseline and follow-up At baseline and follow-up, the absolute HR-HPV positivity was significantly higher in anal samples than in oral samples $(p<0.0001)$ whereas, the percentage of HRHPV detected at anal and oral sites at baseline and at follow-up was comparable $(65.4 \%, 108 / 165$ patients versus $62.4 \%, 103 / 165$ patients, and $9.4 \%, 10 / 106$ patients versus $6.8 \%, 11 / 162$ patients, respectively). The relative frequency of patients with HR-HPV compared to all HPV-positive subjects was significantly higher in anal samples than in oral samples at both baseline $(73.5 \%$, $108 / 147$ patients versus $33.3 \% 10 / 30$ patients $p<0.0001)$ and follow-up (69.6\%, 103/148 patients versus $29.8 \%, 11 /$ 37 patients $p<0.0001)$. A description of the oral and anal swabs results according to HPV detection is reported in Fig. 1.

Overall, 179 anal HR-HPV strains were identified at baseline (strains/person, 1.65) and 194 strains were identified at follow-up (strains/person, 1.88). The most frequent types of HR-HPV detected in the anal swabs at baseline and follow-up were HPV-16 (25.9\%, 28/108, and $28.1 \%, 29 / 103$ of HR-HPV-positive subjects, respectively) and HPV-52 (23.1\%, 25/108 and 27.2\%, 28/103 of HR-HPV positive subjects, respectively).

All HR positive oral samples at baseline and follow-up had a single HR-HPV detection; the prevalence of this pattern was significantly higher compared to the prevalence in the anal site at baseline $(54.6 \%, 59 / 108$ patients $p=0.005)$ and follow-up $(54.4 \%, 56 / 103$ patients, $p=0.002)$. Among these HR-HPV positive anal samples, multiple HRHPV patterns were found in 49 of 108 subjects (45.4\%) at baseline and in 47 of 103 subjects (45.6\%) at follow-up ( $p=0.0331$ with respect to the frequency at baseline) Of note, a different genotype combination was identified in all these patients at baseline and in 90.3\% of these patients at follow-up.

A complete description of the HR-HPV genotypes and of their single and combined detection in the oral and anal swabs is reported in Fig. 2 and Additional file 1.

\section{Relationship between oral and anal results at baseline and follow-up}

A total of 106 subjects at baseline and 162 patients at follow-up had both oral and anal testing results available. In total, the anal results were concordant with the 


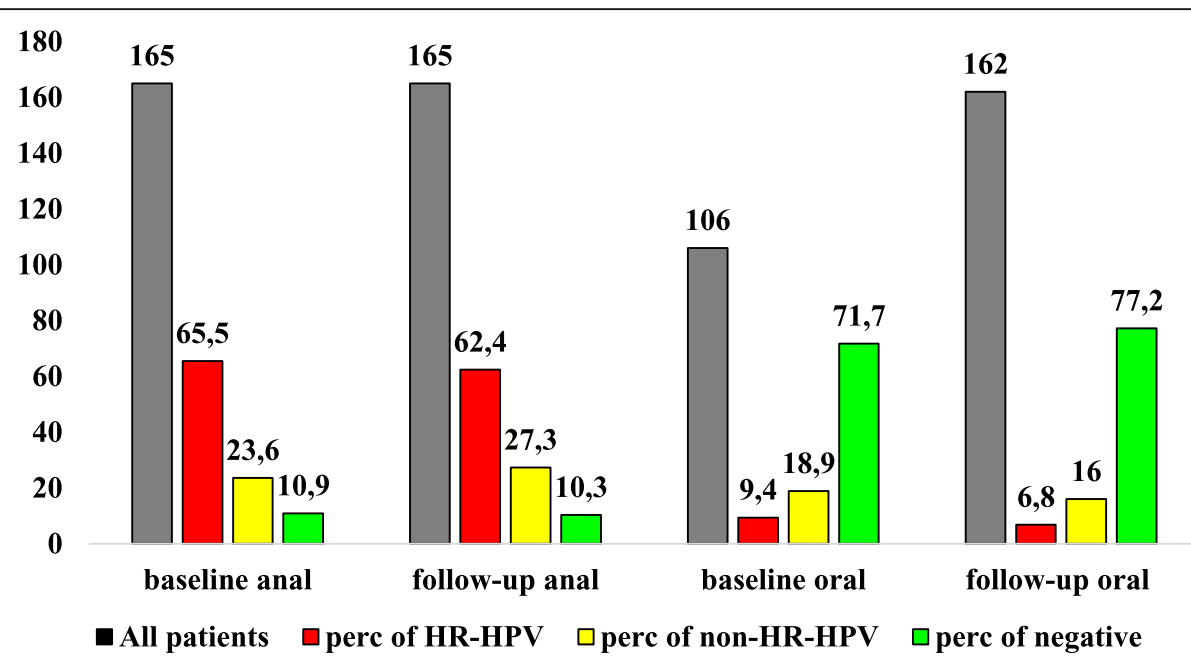

Fig. 1 Description of oral and anal swabs results in MSM at baseline and follow-up (24 months after baseline). Data are expressed as absolute numbers (grey column, corresponding to the total number of samples of the specific study time) and as percentage of samples with HR HPV detection, non-HR HPV detection and no HPV detection in the specific study time (red, yellow and green columns). MSM: men who have sex with men. HR-HPV: high risk HPV genotypes. Non-HR-HPV: only non high risk HPV genotypes. HPV: human papillomavirus

oral results in 17 patients (16\%) at baseline and in 34 patients $(21 \%)$ at follow-up.

The highest rate of concordance between the oral and anal figure was found for negative results $(66.7 \%, 6 / 9 \mathrm{pa}-$ tients at baseline and 100\%, 17/17 patients at follow-up) and the lowest for HR-HPV detection $(9.1 \%, 7 / 77$ patients at baseline, $p=0.0002$ and $7.9 \%, 8 / 101$ patients at follow-up, $p<0.0001$ with respect to the percentage of concordant negative results at the same study time).

Interestingly, almost all patients with HR positivity at both the anal and oral sites had different HR-HPV genotypes; only a single subject had HPV-16 in both samples at baseline. Table 1 summarizes the combinations between the anal and oral results at baseline and followup.

In the subgroup of subjects with all samples tested at both baseline and follow-up, 3 subjects (2.9\%) were always positive for HR-HPV at both the anal and oral sites and 3 patients $(2.9 \%)$ were persistently negative.

\section{Longitudinal changes in oral and anal testing from baseline to follow-up}

A total of 104 patients had HPV tested in oral samples at both baseline and at follow-up. The percentage of patients with the same pattern at both study times was significantly higher in patients with negative results $(85.3 \%$, $64 / 75$ patients) with respect to the detection of non-HRHPV $(36.8 \%, 7 / 19$ patients $p<0.0001)$ and of HR-HPV (40\%, $4 / 10$ patients, $p=0.003)$. The HR genotypes of the 4 patients with confirmed HR-HPV at the oral sites were the same at baseline and follow-up (HPV-16, HPV-35, HPV-56, HPV-59).
The number of patients with anal HR-HPV was comparable at baseline and follow-up (108 and 103 respectively), and most (80 out of $108,74.1 \%$ ) of the subjects with HR-HPV at baseline were also positive for HR-HPV at follow-up ( $p=0.032$ with respect to the $40 \%$ found in oral samples). Patients receiving ART showed a significantly lower frequency of confirmed HR detection compared to untreated patients $(43.3 \%, 52 / 120$ patients versus $62.2 \%, 28 / 45$ patients, $p=0.03)$. At follow-up, 30 out of 80 patients $(37.5 \%)$ had HR-HPV at follow-up with a different genotype than that at baseline, $37 \mathrm{pa}-$ tients $(46.3 \%)$ had one HR-HPV strain with the same genotype at both baseline and follow-up, 11 (13.7\%) had 2 HR-HPV and 2 (2.5\%) had 3 HR-HPV with the same genotypes at baseline and follow-up.

Most patients who become HR-HPV-positive at follow-up reported a single HR positivity: $83.3 \%$ (5/6 patients) in case of negative result at baseline and $82.3 \%$ (14/17 patients) in the case of non-HR-HPV identification at baseline. A detailed description of the oral and anal HR-HPV patterns modifications from baseline to follow-up is reported in Fig. 3.

Fifty-nine subjects had a single type of HR-HPV found at baseline; at follow-up, 6 subjects (10.2\%) became negative, 12 patients (20.3\%) had non-HR-HPV and 41 (69.5\%) had confirmed HR-HPV positivity. Twenty-four of the 41 patients had 1 HR-HPV strain at follow-up (16 subjects with a different genotype from baseline), and 17 (28.8\%) subjects had an increased number of HR-HPV strains detected at follow-up. The percentage of MSM with confirmed HR-HPV detection at follow-up was $80 \%$ in the case of $2 \mathrm{HR}$ found at baseline, $75 \%$ in the case of 3 HR-HPV found at baseline and 100\% when 4 HR-HPV 




(b)

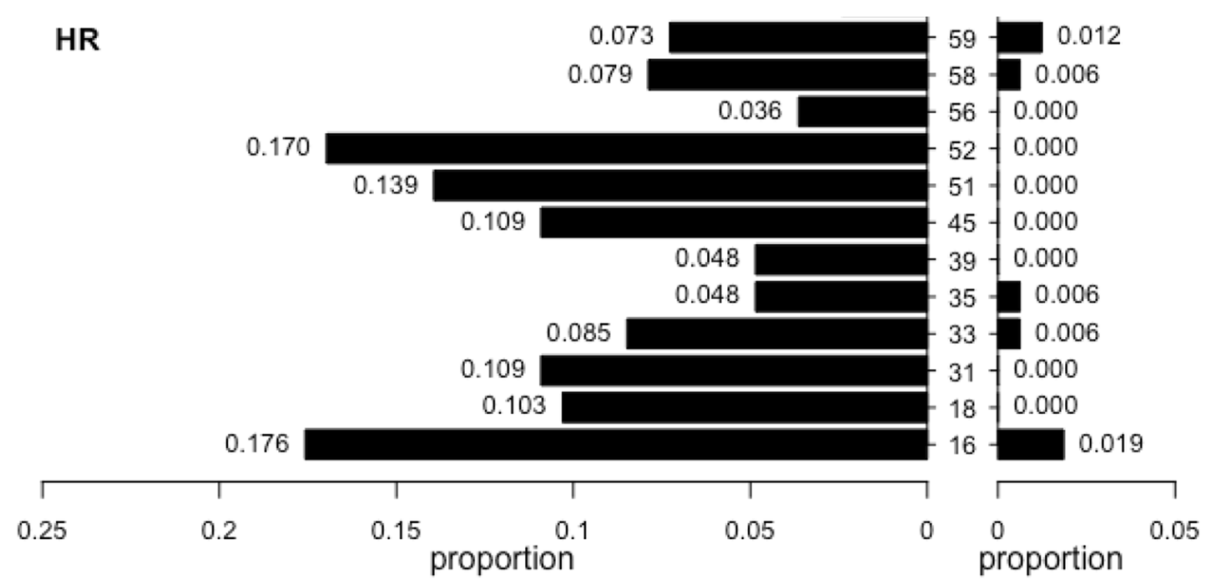

Fig. 2 Description of HR-HPV genotype prevalence at baseline (a) and at follow-up (24 months after baseline) (b). Data corresponding to the anal site are on the left and data corresponding to the oral site are on the right: the prevalence is expressed as proportion respect to the number of subjects with HPV testing available (165 anal samples at baseline and at follow-up, 106 oral samples at baseline and 162 oral samples at follow-up). HR-HPV: high risk HPV genotypes. HPV: human papilloma virus

Table 1 Description of HPV detection in paired anal and oral swabs baseline and at follow-up (24 months after baseline)

\begin{tabular}{llll}
\hline & Baseline oral HR-HPV $n(\%)$ & Baseline oral non-HR-HPV n (\%) & Baseline oral neg $n$ (\%) \\
Baseline anal HR-HPV (77 pts) & $7(9.1)$ & $15(19.5)$ & $55(71.4)$ \\
Baseline anal non-HR HPV (20 pts) & $1(5)$ & $4(20)$ & $15(75)$ \\
Baseline anal neg (9 pts) & $2(22.2)$ & $1(11.1)$ & $6(66.7)$ \\
& Follow-up oral HR-HPV n (\%) & Follow-up oral non-HR-HPV n (\%) & Follow-up oral neg n (\%) \\
Follow-up anal HR-HPV (101 pts) & $8(7.9)$ & $17(16.8)$ & $76(75.2)$ \\
Follow-up anal non-HR-HPV (44 pts) & $3(6.8)$ & $9(20.5)$ & $32(72.7)$ \\
Follow-up anal neg (17 pts) & 0 & 0 & $17(100)$ \\
\hline
\end{tabular}

HR: high risk HPV genotypes

non-HR: only not high risk HPV genotypes

HPV: Human papillomavirus

pts: patients 


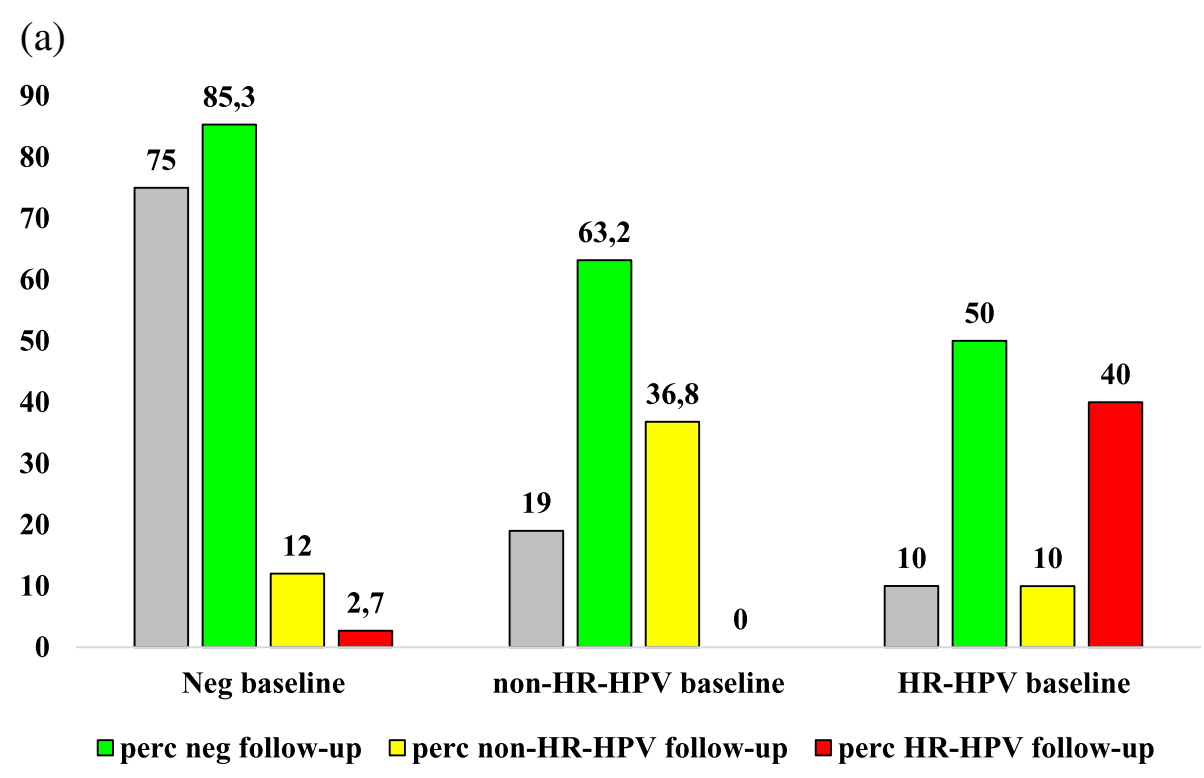

(b)

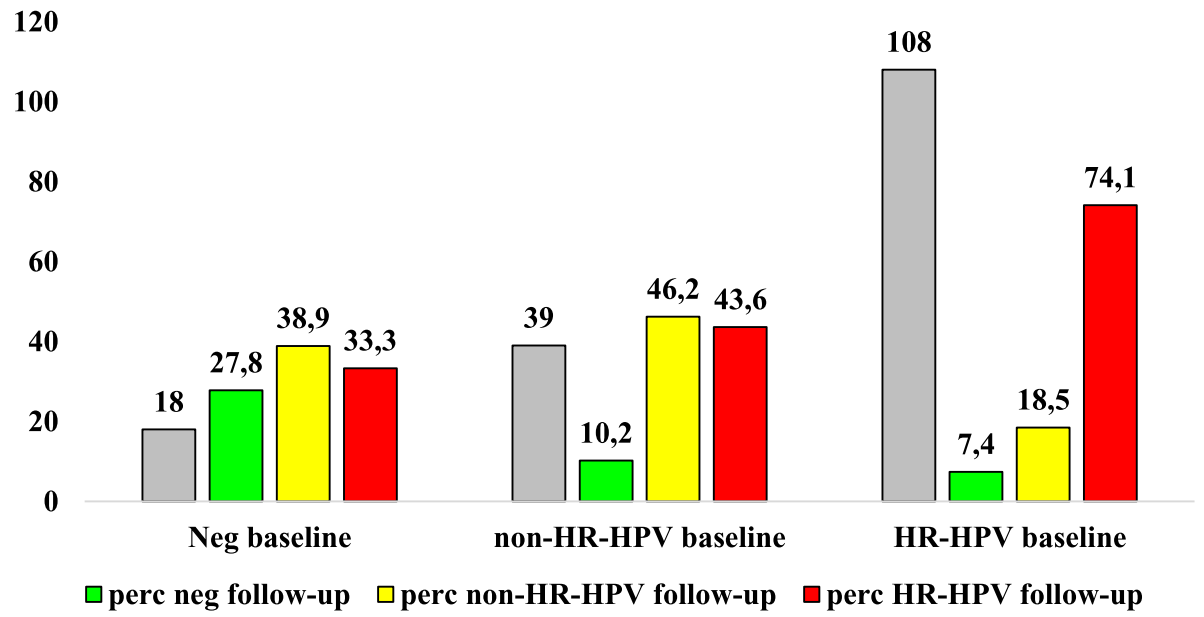

Fig. 3 Description of modifications at follow-up (24 months after baseline) of HPV patterns detected at baseline. The first column correspond to baseline data (expressed as absolute number). Green, yellow and red columns correspond to the proportion of negative, non-HR-HPV HR-HPV respectively detected at follow-up as evolution of each HPV pattern at baseline. Panel a refers to oral samples and panel $\mathbf{b}$ refers to anal samples. HR-HPV: HR HPV genotypes. Non-HR-HPV: only non high risk HPV genotypes

were found at baseline (only 3 patients were included in the subgroup with 4 HR genotypes); no patients with $\geq 3$ types of HR-HPV at baseline had a negative result at follow-up (Table 2).

HPV 16 was the most frequently detected genotype at both baseline and follow-up: no significant difference in the prevalence of the specific HR-HPV genotype at baseline and follow-up was found, and the percentages ranged from 3.03 to $16.97 \%$ at baseline and from 3.64 to $17.58 \%$ at follow-up. A concordant positivity of the same genotype at both time points was reported for HPV-16 ( $p<$ $0.001)$, HPV-18 $(p<0.001)$, HPV-31 $(p=0.000)$, HPV- $35(p=0.05), \mathrm{HPV}-45(p=0.000), \mathrm{HPV}-51(p=0.000)$, HPV-58 $(p=0.004)$ and HPV-59 $(p=0.043)$.

HPV infection by genotypes covered by the nonavalent vaccine

Overall, 7 of the $16 \mathrm{HPV}$ infections detected in oral sites at baseline and follow-up were found to be caused by HR-HPV strains that were covered by the vaccine. Only 1 patient had HPV16 detected at both study times.

Nearly half of the patients with HR-HPV positivity at the anal site $(54.6 \%, 59 / 108$ patients at baseline and $50.5 \%, 52 / 103$ patients at follow-up) had only HR-HPV 
Table 2 Description of HR-HPV evolution from baseline to follow-up (24 months after baseline) according to the number of HR-HPV detected at baseline. The results obtained at follow-up are reported as absolute number and relative percentage within the specific subgroup

\begin{tabular}{|c|c|c|c|c|c|c|c|}
\hline & \multirow{3}{*}{$\begin{array}{l}\text { Total number } \\
\text { at baseline }\end{array}$} & \multicolumn{6}{|c|}{ Follow-up } \\
\hline & & Neg & non-HR & $1 \mathrm{HR}-\mathrm{HPV}$ & $2 \mathrm{HR}-\mathrm{HPV}$ & 3 HR-HPV & $\geq 4 \mathrm{HR}-\mathrm{HPV}$ \\
\hline & & n (\%) & n (\%) & n (\%) & n (\%) & n (\%) & n (\%) \\
\hline 1 HR-HPV at baseline & 59 & $6(10.2)$ & $12(20.3)$ & $24(40.7)$ & $7(11.9)$ & $8(13.5)$ & $2(3.4)$ \\
\hline 2 HR-HPV at baseline & 30 & $2(6.7)$ & $4(13.3)$ & $9(30)$ & $4(13.3)$ & $6(20)$ & $5(16.7)$ \\
\hline 3 HR-HPV at baseline & 16 & 0 & $4(25)$ & $4(25)$ & $1(6.3)$ & $5(31.2)$ & $2(12.5)$ \\
\hline 4 HR-HPV at baseline & 3 & 0 & 0 & 0 & $2(66.7)$ & $1(33.3)$ & 0 \\
\hline
\end{tabular}

HR: high risk HPV genotypes

non-HR: only not high risk HPV genotypes

genotypes covered by the vaccine, and 25 (15.2\%) of the 165 subjects had this result at both study time points. The median age of the 25 patients was 44 years (range $39-55$ years), and the nadir median value was $350 \mathrm{CD} 4+$ cells $/ \mathrm{mm}^{3}$ (range $26-535 \mathrm{CD} 4+$ cells $/ \mathrm{mm}^{3}$ ). Furthermore, single HR-HPV detection was the more frequent result (16 patients at baseline and 17 patients at followup). A detailed description of the HR-HPV genotypes identified at baseline and follow-up is reported in Fig. 4. Non-HR-HPV covered by the vaccine was not detected at the oral site while at anal site 5 patients were positive for HPV-6 (4 patients both at baseline and follow-up and one subject only at follow-up) and one patient was positive for HPV-11 at baseline and at follow-up.

\section{Discussion}

This study focused on the characteristics of persistence and pattern evolution of HR-HPV infection in oral and anal areas over time in a cohort of HIV-positive MSM tested over a 24-month interval. We selected this study duration because the spontaneous clearance of nearly all HR-HPV strains occurs within this interval [16]; thus, we could identify the persistence of a specific HPV genotype and/or genotype patterns over time even if we cannot rule out possible reinfection.

In our opinion, HPV vaccination should be recommended for all HIV-positive MSM, regardless of age and of HPV detection at any site, even if published data are scarce, because of the high incidence of anal cancer in these subjects [17]. Subjects who remain HR-HPVnegative are the priority for vaccination, particularly if the negative result was confirmed over time and in more than one site, as in our small cohort of HIV-positive MSM (2.9\% of all the patients included in this study). The European AIDS Clinical Society guidelines 2018 state that if HPV infection is established, the efficacy of the vaccine is questionable [10]; however, the role of a vaccine is to prevent infection, and HPV has the ability

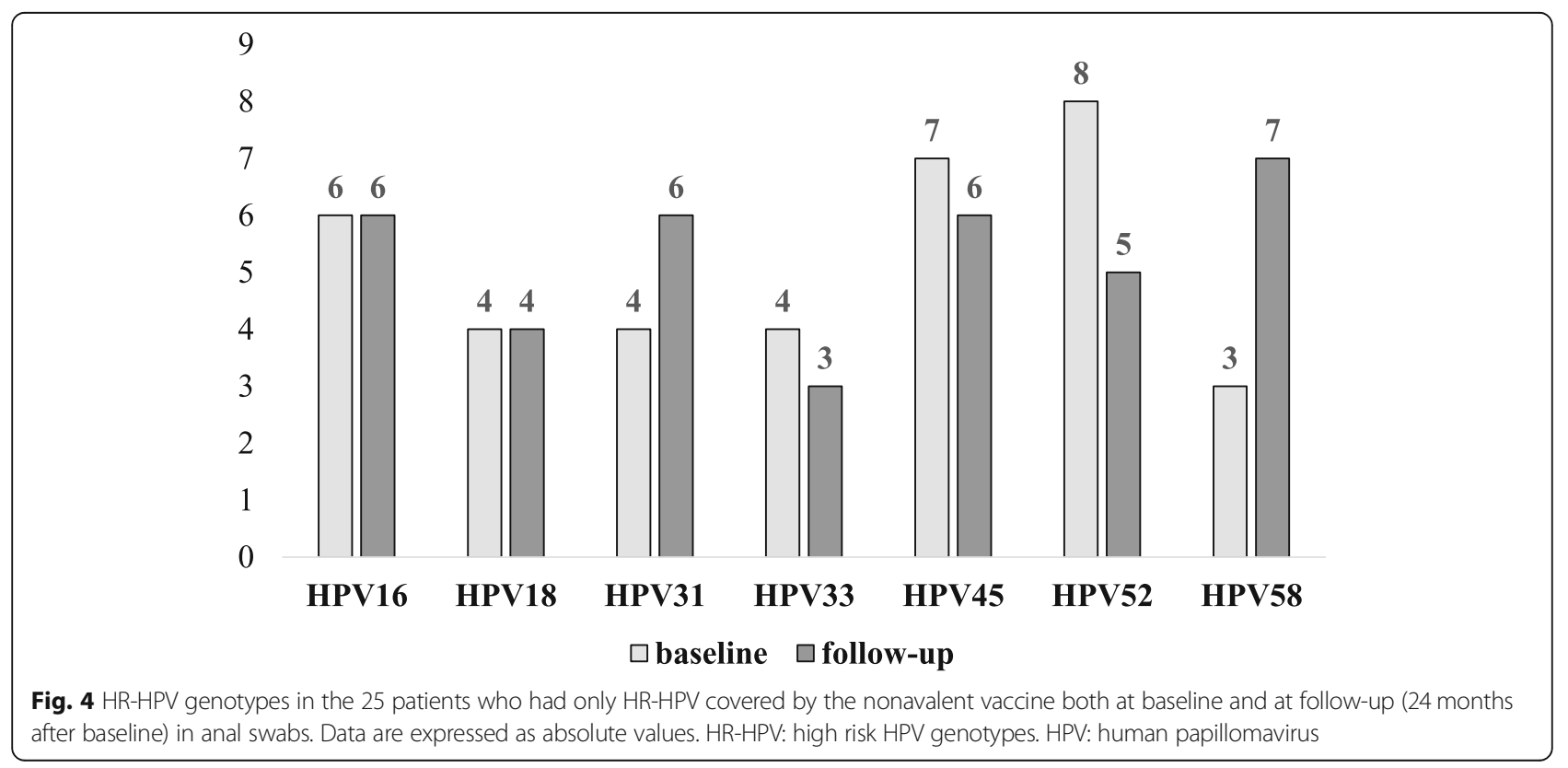


to be responsible for multiple infections. Therefore, the concept of established infection could potentially be applied to each HPV genotype. Furthermore, available HPV vaccines are safe and tolerable [18-20] and a persistent cross-reactive immune response against HR-HPV not covered by the vaccine was shown in vaccinated women [21]. However, vaccination has a clinically relevant role also in subjects with established HPV infection $[13,22]$. We analysed the HR-HPV patterns in HIVpositive MSM retained in care in a tertiary level hospital of a high-income country that provides free care and therapy, which is the best clinical scenario. Based on our results, we believe that HPV vaccination should be prescribed in this clinical setting because all the tools that can reduce the risk of cancer should be used [23]. However, HPV vaccination prescription in different cohort of HIV+ MSM can be impeded by social and economical barriers [24].

The first result of our study was a significantly higher frequency of all HPV genotypes and of confirmed HRHPV detection at the anal site at follow-up compared to the oral site (overall $81.8 \%$ versus 11.6 and $74.1 \%$ versus $40 \%$ for HR-HPV genotypes). Beachler et al. [25] reported the same difference in a cohort of 404 HIV subjects: (69 MSM): these data referred to any nononcogenic and oncogenic types; no specific analysis on HR was reported. Limited data concerning HR-HPV persistence in patients for whom more than a single site was tested have been reported. Parisi et al. [1] described a persistence rate of $71.4 \%$ in the anal site with no data regarding the oral site in 166 HIV+ MSM studied with a 6 -month time interval between the 2 samplings. These authors demonstrated that any HR-HPV genotype detection in anal samples at either time point was positively related to a higher HIV plasma viral load and negatively related to ongoing ART. We investigated the role of plasma HIV viremia suppression on HR-HPV confirmed detection and found that the percentage of confirmed HR detection at both baseline and follow-up (2 years later) in this site was lower in patients receiving successful ART. The influence of ongoing plasma HIV viremia on HR-HPV detection over time has previously been studied with different approaches. Geskus et al. [26] described a lower HR-HPV clearance rate in MSM with higher plasma HIV RNA (relative to 1000 copies/ml) in a median time interval between measurements of 1.1 years, and Wiley et al. [27] demonstrated that the prevalence of HR-HPV was lower for every $10 \%$ increase in the number of study visits in which HIV RNA measured $<50$ copies/mL during the study period. We could not evaluate the role of the HIV viral load in HR persistence at the oral site, possibly because of the low numerosity of the HR-HPV identified. The breakdown of the mucosal epithelial barrier by HIV may explain this association:
Nazli et al. [28] demonstrated that exposure to HIV-1 led to an upregulation of inflammatory cytokine production and to a significant decrease in tight junction protein expression and increased permeability while Tugizov et al. [29] showed that tight junction disruption of the oral and anal epithelium due to the expression of the HIV tat and gp120 increased HPV pseudovirion paracellular penetration into the epithelium. However, we cannot exclude an independent role for immuneactivation, as described for the interplay between HIV and CMV, EBV, and HHV-8 [30, 31].

In our study, the anal HR-HPV pattern was more complex than the oral pattern, both at baseline and at follow-up. The influence of the anatomic site of infection on the natural history of HPV is multifaceted and can include a faster clearance from oral epithelia and the continuous flow of saliva and associated immunoglobulins in the oral region [32, 33]. Papasavvas et al. [34] hypothesized a relationship between HR-HPV infection at the cervical site and increased immune activation in HIV infected women who were on successful ART, confirming the activating role of a viral co-infection previously reported for HCV in HIV patients with a virologic response [35]. This mechanism can contribute to the higher HR-HPV complexity (i.e., increased number of HR-HPV genotypes) at the anal site, which is rich in lymphoid tissue [36].

Almost all patients with HR-HPV positivity at both the anal and oral sites had different HR-HPV genotypes, as previously described [37, 38]. These results indicate the importance of testing a high-risk population in multiple sites: a single time point or single district data offered partial information both on HR-HPV detection (yes or no) and on possible multiple HPV detection in the specific patient. We found that only $2.9 \%$ of the subjects with oral and anal testing available were free from any HPV genotype and that $28.8 \%$ of the patients with one genotype of HR-HPV detected at baseline had an increased number of HR genotypes at follow-up. Based on this finding, we could speculate that almost all HIV+ MSM will become HPV positive during life and HPV detection could be missed or under evaluated with insufficient follow-up. Furthermore, two recent works $[39,40]$ described an association between multiple HPV infections and low-grade or high-grade squamous intraepithelial lesions at the anal site in HIV-positive men. Notably, the patients included in the work by Rovelli et al. [39] had no clinical symptoms. The presence of only HR-HPV genotypes covered by the nonavalent vaccine was demonstrated in nearly half of the patients at the oral and anal level. Therefore, an extended vaccination programme could reduce the increased complexity at the anal site and the risk of the first HR-HPV acquisition at the oral site [41]. 
During all DNA preparation, extraction and purification steps, precautions were taken to reduce the risk of false positive results or cross-contamination. However we cannot exclude a contamination. Besides this one, other limitations of this study must be recognized. First, we only sampled patients at 2 time points; thus, the evidence of persistent HR-HPV infection may instead be the clearance and subsequent transient exposure to the same genotype prior to the second sampling. Our HRHPV detection was according to the type level; therefore, we could not exclude the possibility that someone could become infected with another variant of the same HRHPV type; however, the young age of the subjects does not seem to indicate a modification in the exposure to risk. Second, only a small group of patients had all 4 samples available for testing, and positive HR detection was low at the oral site. These limitations restricted the power of the statistical analysis to detect correlations. Third, a more complete analysis on other sites of infection, such as the penis, and information about partners would make the analysis more informative. Fourth, we did not collect data on the patients' sexual behaviour. Instead, our study focused on modifications of HR-HPV patterns after a 48-month study interval in adult patients rather than the reasons behind the modifications. Fifth, many oral samples were not evaluated because they were negative for beta globin, which indicates low cellularity; a different sampling method (i.e., toothbrush sampling) [42] could have helped increase the number of valid samples.

\section{Conclusions}

In conclusion, HR-HPV detection is a clinical problem for almost all HIV positive MSM and requires careful surveillance. The anal HR-HPV pattern was more complex than the oral pattern at both baseline and followup. Effective ART seems to have a positive impact on HR-HPV confirmed detection at the anal level and should be associated with an extended vaccine campaign in light of ongoing new HIV diagnoses mostly in young patients $[43,44]$.

\section{Additional file}

Additional file 1: Description of HR-HPV genotypes in oral and anal samples at baseline and at follow-up ( 24 months after baseline). Data are reported according to the number of HR-HPV genotypes detected and to the relative frequency of each specific combination within the group. (PDF $162 \mathrm{~kb}$ )

\section{Abbreviations}

ART: Antiretroviral therapy; HPV: Human papillomavirus; HR-HPV: High-risk HPV; MSM: Men who have sex with men; non-HR-HPV: Only not high risk HPV genotypess; PBS: Phosphate-buffered saline; vax-HPV: HR-HPV covered by the nonavalent HPV vaccine

\section{Acknowledgements}

SGP, MB, RS, MC are members of CAVeAT (Cohort of Amici Venetian for Antiretroviral Treatment).

A professional language editing was made.

\section{Authors' contributions}

SGP designed and coordinated the study, supervised the laboratory experiments, collected the data, interpreted the findings and wrote the paper. MB helped to design the study, performed the statistical analysis and wrote the paper. RS helped to design the study and to interpret the findings, managed the patients, and collected the samples. SA performed the laboratory experiments, helped to interpret the findings. CM performed the statistical analysis. MC collaborated to the statistical analysis and drafted the work. CDV collaborated to laboratory experiments and drafted the work. NM performed the laboratory experiments. DZ collaborated to laboratory experiments and to interpretation of data. LS helped to write the paper and substantively revised it. MA helped to interpret the findings and write the paper. GP helped to interpret the findings and write the paper. All authors read and approved the final manuscript.

\section{Funding}

The work was supported by MURST ex 60\% 2015 (to M.B.). The funders had no role in the study design, collection, analysis or interpretation of the data writing the manuscript, or the decision to submit the paper for publication.

\section{Availability of data and materials}

The datasets generated and analysed for this study are not publicy available because they include clinical data.

\section{Ethics approval and consent to participate}

The Ethics Committee of Padova University Hospital (prot. 2606-12P) approved this study, which was made according to the Declaration of Helsinki and local legislation. The patients included in the study gave written informed consent to all procedures and they agreed with the anonymous use of their data for scientific aims and publication.

\section{Consent for publication}

Not applicable.

\section{Competing interests}

SGP: had received speaker fees and research funding from Gilead Sciences, ViiV Healthcare, Abbott, Abbvie, Janssen, Merck Sharp \& Dohme.

MB: had received speaker fees from Gilead Sciences.

L.S. had received travel grants from Gilead Sciences and Merck Sharp \& Dohme.

MA: had received speaker fees, travel grants, and consulting fees from Abbvie, Bristol-Myers Squibb, Gilead Sciences, ViiV Healthcare, and JanssenCilag.

Other Authors declared they have no competing interests.

\section{Author details}

1Department of Molecular Medicine, University of Padova, Via Gabelli 63 35100 Padova, Italy. ${ }^{2}$ Infectious Diseases Unit, Padova Hospital, Via Giustiniani, 2 -, 35128 Padova, Italy. ${ }^{3}$ Center of Diffusive Diseases, ULSS 9, Via Campania 1, 37136 Verona, Italy. ${ }^{4}$ Clinical Infectious Diseases, Tor Vergata University, Viale Oxford, 81, 00133 Rome, Italy.

Received: 24 January 2019 Accepted: 17 May 2019

Published online: 28 May 2019

\section{References}

1. Parisi SG, Cruciani M, Scaggiante R, Boldrin C, Andreis S, Dal Bello F, et al. Anal and oral human papillomavirus (HPV) infection in HIV-infected subjects in northern Italy: a longitudinal cohort study among men who have sex with men. BMC Infect Dis. 2011;11:150.

2. Combes JD, Heard I, Poizot-Martin I, Canestri A, Lion A, Piroth L, et al. Prevalence and risk factors for anal human papillomavirus infection in human immunodeficiency virus-positive men who have sex with men. J Infect Dis. 2018;217:1535-43. 
3. King EM, Oomeer S, Gilson R, Copas A, Beddows S, Soldan K, et al. Oral human papillomavirus infection in men who have sex with men: a systematic review and meta-analysis. PLoS One. 2016;11:e0157976.

4. Mooij SH, Boot HJ, Speksnijder AG, Stolte IG, Meijer CJLM, Snijders PJF, et al. AIDS. 2013;27:2117-28.

5. Marra E, Siegenbeek van Heukelom ML, Leeman A, Waterboer T, Meijer CJLM, Snijders PJF, et al. Virological and serological predictors of anal highgrade squamous intraepithelial lesions among HIV-positive men who have sex with men. Clin Infect Dis. 2019;68:1377-87.

6. Beachler DC, Weber KM, Margolick JB, Strickler HD, Cranston RD, Burk RD, et al. Risk factors for oral HPV infection among a high prevalence population of HIV-positive and at-risk HIV-negative adults. Cancer Epidemiol Biomark Prev. 2012;21:122-33.

7. Gravitt PE. The known unknowns of HPV natural history. J Clin Invest. 2011; 121:4593-9.

8. Vergori A, Garbuglia AR, Piselli P, Del Nonno F, Sias C, Lupi F, et al. Oral human papillomavirus DNA detection in HIV-positive men: prevalence, predictors, and co-occurrence at anal site. BMC Infect Dis. 2018;18:25.

9. Blas MM, Brown B, Menacho L, Alva IE, Silva-Santisteban A, Carcamo C. HPV prevalence in multiple anatomical sites among men who have sex with men in Peru. PLoS One. 2015;10:e0139524.

10. European AIDS Clinical Society Guidelines, Version 6.1. Accessed 17 Oct 2018.//www.eacsociety.org

11. Grennan JT, Loutfy MR, Su D, Harrigan PR, Cooper C, Klein M, et al. Magnitude of virologic blips is associated with a higher risk for virologic rebound in HIV-infected individuals: a recurrent events analysis. J Infect Dis. 2012;205:1230-8

12. Basso M, Franzetti M, Scaggiante R, Sattin A, Mengoli C, Cruciani M, et al. HCV RNA viral load is independent from CD4 cell count and plasma HIV RNA viral load in immunocompetent HIV-HCV co-infected patients: a 3-years follow-up study. AIDS Res Ther. 2014;11:21.

13. Foresta C, Garolla A, Parisi S, Ghezzi M, Bertoldo A, Di Nisio A, et al. HPV prophylactic vaccination in males improves the clearance of semen infection. EBioMedicine. 2015:2:1487-93.

14. IARC Working Group on the Evaluation of Carcinogenic Risks to Humans. Biological agents. Volume 100 B. a review of human carcinogens. IARC Monogr Eval Carcinog Risks Hum. 2012;100(Pt B):1-441.

15. European Medicines Agency (2015) Gardasil 9. Summary of product characteristics

16. Giuliano AR, Lee JH, Fulp W, Villa LL, Lazcano E, Papenfuss MR, et al. Incidence and clearance of genital human papillomavirus infection in men (HIM): a cohort study. Lancet. 2011;377:932-40.

17. Martínez-Gómez X, Curran A, Campins M, Alemany L, Rodrigo-Pendás JÁ, Borruel N, et al. Multidisciplinary, evidence-based consensus guidelines for human papillomavirus (HPV) vaccination in high-risk populations, Spain, 2016 Euro Surveill. 2019:24. https://doi.org/10.2807/1560-7917.ES.2019.24.7.1700857.

18. GlaxoSmithKline. Cervarix suspension for injection: summary of product characteristics. GlaxoSmithKline; 2012. Available from:http://www.ema. europa.eu/docs/en_GB/document_library/EPAR_-_Product_Information/ human/000721/WC500024632.pdf</eref>. Accessed 18 Apr 2019.

19. M Sharp \& Dohme BV. Gardasil suspension for injection: summary of product characteristics. Merck Sharp \& Dohme BV; 2011. Available from: http://www ema.europa.eu/docs/en_GB/document_library/EPAR_-_Product_Information/ human/000703/WC500021142.pdf</eref>. Accessed 18 Apr 2019.

20. M Sharp \& Dohme BV. Gardasil 9 suspension for injection: summary of product characteristics. Merck Sharp \& Dohme BV; 2015. Available from: http://www.ema.europa.eu/docs/en_GB/document_library/EPAR_-_Product_ Information/human/003852/WC500189111.pdf. Accessed 18 Apr 2019.

21. Folschweiller $N$, Behre U, Dionne M, Durando P, Esposito S, Ferguson L, et al. Long-term cross-reactivity against nonvaccine human papillomavirus types 31 and 45 after 2- or 3-dose schedules of the AS04-Adjuvanted human HPV-16/18 vaccine. J Infect Dis 2019. doi: https://doi.org/10.1093/ infdis/jiy743. [Epub ahead of print].

22. Rosenberg T, Philipsen BB, Mehlum CS, Dyrvig AK, Wehberg S, Chirilǎ M, et al. Therapeutic use of the human papillomavirus vaccine on recurrent respiratory papillomatosis: a systematic review and meta-analysis. J Infect Dis. 2019;219:1016-25.

23. Ong JJ, Walker S, Grulich A, Hoy J, Read TRH, Bradshaw C, et al. Incidence, clearance, and persistence of anal human papillomavirus in men who have sex with men living with human immunodeficiency virus: implications for human papillomavirus vaccination. Sex Transm Dis. 2019;46:229-33.
24. Galea JT, Monsour E, Nureña CR, Blas MM, Brown B. HPV vaccine knowledge and acceptability among Peruvian men who have sex with men and transgender women: a pilot, qualitative study. PLoS One. 2017;12:e0172964.

25. Beachler DC, D'Souza G, Sugar EA, Xiao W, Gillison ML. Natural history of anal vs oral HPV infection in HIV-infected men and women. J Infect Dis. 2013:208:330-9.

26. Geskus RB, González C, Torres M, Del Romero J, Viciana P, Masiá M, et al. Incidence and clearance of anal high-risk human papillomavirus in HIV-positive men who have sex with men: estimates and risk factors. AIDS. 2016;30:37-44.

27. Wiley DJ, Li X, Hsu H, Seaberg EC, Cranston RD, Young S, et al. Factors affecting the prevalence of strongly and weakly carcinogenic and lower-risk human papillomaviruses in anal specimens in a cohort of men who have sex with men (MSM). PLoS One. 2013:8:e79492.

28. Nazli A, Chan O, Dobson-Belaire WN, Ouellet M, Tremblay MJ, Gray-Owen $\mathrm{SD}$, et al. Exposure to HIV-1 directly impairs mucosal epithelial barrier integrity allowing microbial translocation. PLoS Pathog. 2010;6:e1000852.

29. Tugizov SM, Herrera R, Chin-Hong P, Veluppillai P, Greenspan D, Michael Berry J, et al. HIV-associated disruption of mucosal epithelium facilitates paracellular penetration by human papillomavirus. Virology. 2013;446:378-88.

30. Scaggiante $R$, Andreis S, Basso M, Franchin E, Franzetti M, Del Vecchio C, et al. Epstein-Barr and cytomegalovirus DNA salivary shedding correlate with long-term plasma HIV RNA detection in HIV-infected men who have sex with men. J Med Virol. 2016;88:1211-21.

31. Basso M, Andreis S, Scaggiante R, Franchin E, Zago D, Biasolo MA, et al. Cytomegalovirus, Epstein-Barr virus and human herpesvirus 8 salivary shedding in HIV positive men who have sex with men with controlled and uncontrolled plasma HIV viremia: a 24-month longitudinal study. BMC Infect Dis. 2018;18:683

32. Steinau M, Gorbach P, Gratzer B, Braxton J, Kerndt PR, Crosby RA, et al. Concordance between anal and Oral human papillomavirus infections among Young men who have sex with men. J Infect Dis. 2017;215:1832-5.

33. Pickard RK, Xiao W, Broutian TR, He X, Gillison ML. The prevalence and incidence of oral human papillomavirus infection among young men and women, aged 18-30 years. Sex Transm Dis. 2012;39:559-66.

34. Papasavvas E, Surrey LF, Glencross DK, Azzoni L, Joseph J, Omar T, et al. High-risk oncogenic HPV genotype infection associates with increased immune activation and T cell exhaustion in ART-suppressed HIV-1-infected women. Oncoimmunology. 2016;5:e1128612.

35. Parisi SG, Andreis S, Mengoli C, Menegotto N, Cavinato S, Scaggiante R, et al. Soluble CD163 and soluble CD14 plasma levels but not cellular HIV-DNA decrease during successful interferon-free anti-HCV therapy in HIV-1-HCV co-infected patients on effective combined anti-HIV treatment. Med Microbiol Immunol. 2018;207:183-94.

36. Telwatte $\mathrm{S}$, Lee $\mathrm{S}$, Somsouk M, Hatano H, Baker C, Kaiser $\mathrm{P}$, et al. Gut and blood differ in constitutive blocks to HIV transcription, suggesting tissuespecific differences in the mechanisms that govern HIV latency. PLoS Pathog. 2018;14:e1007357.

37. De Vuyst H, Clifford GM, Nascimento MC, Madeleine MM, Franceschi S. Prevalence and type distribution of human papillomavirus in carcinoma and intraepithelial neoplasia of the vulva, vagina and anus: a meta-analysis. Int J Cancer. 2009;124:1626-36.

38. Chuang E, Lim E, Milne C, Zhu X, Agsalda M, Killeen J, et al. Human papillomavirus at multiple sites associated with anal squamous intraepithelial lesions in HIV-seropositive individuals. Ann Clin Cytol Pathol. 2016;2.

39. Rovelli C, Poli A, Galli L, Cernuschi M, Tamburini AM, Racca S, et al. Presence of multiple genotypes in subjects with HPV-16 infection is highly associated with anal squamous intraepithelial lesions in HIV-1 infected males. PLoS One. 2017:12:e0186367.

40. Cheng SH, Liao KS, Wang CC, Cheng CY, Chu FY. Multiple types of human papillomavirus infection and anal precancerous lesions in HIV-infected men in Taiwan: a cross-sectional study. BMJ Open. 2018;8:e019894

41. Riethmuller D, Jacquard AC, Lacau St Guily J, Aubin F, Carcopino X, Pradat P, et al. Potential impact of a nonavalent HPV vaccine on the occurrence of HPV-related diseases in France. BMC Public Health. 2015;15:453.

42. Ong JJ, Read T, Chen M, Walker S, Law M, Bradshaw C, et al. Improving oral human papillomavirus detection using toothbrush sampling in HIV-positive men who have sex with men. J Clin Microbiol. 2014;52:2206-9.

43. Parisi SG, Andreis S, Scaggiante R, Cruciani M, Ferretto R, Manfrin V, et al. Decreasing trends of drug resistance and increase of non-B subtypes 
amongst subjects recently diagnosed as HIV-infected over the period 20042012 in the Veneto region, Italy. J Glob Antimicrob Resist. 2013;1:201-6.

44. Andreis $S$, Basso M, Scaggiante R, Cruciani M, Ferretto R, Manfrin V, et al.

Drug resistance in $B$ and non- $B$ subtypes amongst subjects recently

diagnosed as primary/recent or chronic HIV-infected over the period 2013-

2016: impact on susceptibility to first-line strategies including integrase

strand-transfer inhibitors. J Glob Antimicrob Resist. 2017;10:106-12.

\section{Publisher's Note}

Springer Nature remains neutral with regard to jurisdictional claims in published maps and institutional affiliations.

Ready to submit your research? Choose BMC and benefit from:

- fast, convenient online submission

- thorough peer review by experienced researchers in your field

- rapid publication on acceptance

- support for research data, including large and complex data types

- gold Open Access which fosters wider collaboration and increased citations

- maximum visibility for your research: over $100 \mathrm{M}$ website views per year

At $\mathrm{BMC}$, research is always in progress.

Learn more biomedcentral.com/submissions 